\title{
Pemphigus erythematosus relapse associated with atorvastatin intake
}

\author{
This article was published in the following Dove Press journal: \\ Drug Design, Development and Therapy \\ 18 September 2014 \\ Number of times this article has been viewed
}

\author{
Ada Lo Schiavo' \\ Rosa Valentina Puca' \\ Francesca Romano' \\ Roberto Cozzi ${ }^{2}$ \\ 'Department of Dermatology, Second \\ University of Naples, Naples, Italy; \\ 2Department of Dermatology, AORN \\ "A Cardarelli", Naples, Italy
}

\begin{abstract}
Statins, also known as 3-hydroxy-3-methylglutaril-CoA reductase inhibitors, are well-tolerated drugs used for prevention of atherosclerosis and cardiovascular events. Although they are generally considered safe, some serious adverse effects, such as myositis, myopathy, and rhabdomyolysis can rarely occur. Furthermore, recent data from long-term follow-up on patients who have been taking statins for a long period of time suggest that prolonged exposure to statins may trigger autoimmune reactions. The exact mechanism of statin-induced autoimmune reactions is unclear. Statins, as proapoptotic agents, release nuclear antigen into the circulation and may induce the production of pathogenic autoantibodies. Herein we report the case of a 70 year-old man who developed a relapse of pemphigus erythematosus, a syndrome with features of both lupus erythematosus and pemphigus, after atorvastatin intake.
\end{abstract}

Keywords: pemphigus erythematosus, autoimmune disease, treatment, pathogenesis, statins

\section{Introduction}

Pemphigus erythematosus (PE) was described for the first time in 1926 by Senear and Usher as a syndrome with features of both lupus erythematosus and pemphigus. ${ }^{1}$ Since the original reports there has been a debate on whether PE is an overlap syndrome or simply a localized variant of pemphigus foliaceus. In fact, clinical and histological findings are similar to those of pemphigus foliaceus. Erythematous scaly lesions over the nose and cheeks in a butterfly distribution simulate cutaneous lupus erythematosus or seborrhoeic dermatitis. Sunlight may exacerbate the disease. Lesions on the trunk, either localized or generalized, are similar to those in pemphigus foliaceus. The antibodies recognize the pemphigus foliaceus antigen, desmoglein 1; in addition, immunoglobulin $\mathrm{G}(\mathrm{IgG})$ and $\mathrm{C} 3$ deposition on cell surface of keratinocytes can be revealed in a group of patients. A positive antinuclear antibody (ANA) is detected in $30 \%-80 \%$ of PE patients but only a few patients have been reported to have PE and lupus erythematosus concurrently. ${ }^{2,3}$ As for pemphigus vulgaris, several exogenous factors are able to induce PE in genetically predisposed individuals, including drug intake, viral infections, exposure to physical agents, and others. Among these factors, drugs are the most common ones, as numerous reports of drug-induced pemphigus show. ${ }^{4}$

Herein we report the case of a 70-year-old man who developed a relapse of PE that had been in remission for 10 years. The recurrence of the disease occurred after the intake of atorvastatin, a potent second-generation statin. On the basis of the close relationship between the onset of cutaneous lesions and statin administration, a diagnosis of pemphigus relapse induced by atorvastatin was suspected. Statins have been associated with several drug-induced autoimmune reactions but, to the best of
Correspondence: Ada Lo Schiavo Department of Dermatology, Second University of Naples, Via Sergio Pansini 5, 80I3I Naples, Italy Tel $+3908 \mid 5666832$

Fax +390815666832

Email ada.loschiavo@unina2.it 
our knowledge, no case of induction of pemphigus has been described in literature until now.

\section{Case report}

In September 2004 a 60-year-old man presented for the first time to the Department of Dermatology, Second University of Naples with erythematous scaly plaques which involved the cheeks in a butterfly distribution symmetrically and crusted lesions localized on the upper part of the chest. Skin biopsy showed intraepithelial superficial blister; Tzanck test revealed acantholytic cells with rare dyskeratotic changes and leukocytes; direct immunofluorescence on perilesional skin showed deposits of IgG and C3 on keratinocytes' surface and along the dermo-epidermal junction. Laboratory screening showed a high titer of anti-desmoglein 1 antibodies (89 U/ $\mathrm{mL})$ and low titer of anti-desmoglein 3 antibodies $(11 \mathrm{U} / \mathrm{mL})$. ANA test was positive at the titer of 1:180, complement levels were normal. On the basis of these findings a diagnosis of PE was made. Systemic therapy with corticosteroids and azathioprine combined with topical treatment with clobetasol propionate resulted in a remission of cutaneous lesions within 3 months. For 6 months, the patient took only azathioprine as maintenance therapy, then all drugs were suspended. The patient remained healthy until October 2013 when he returned to our department for the appearance of numerous erosions and crusted lesions involving seborrheic areas of the chest (Figure 1). Mucous membranes were diseasefree. A recurrence of PE was hypothesized; confirmation of diagnosis was made by routine histopathology and both direct and indirect immunofluorescence microscopy. ANA level was increased (1:320) compared to the level during previous hospitalization. The clinical history disclosed that the patient had been taking atorvastatin for 3 months to treat a severe hypercholesterolemia. He was not taking any other

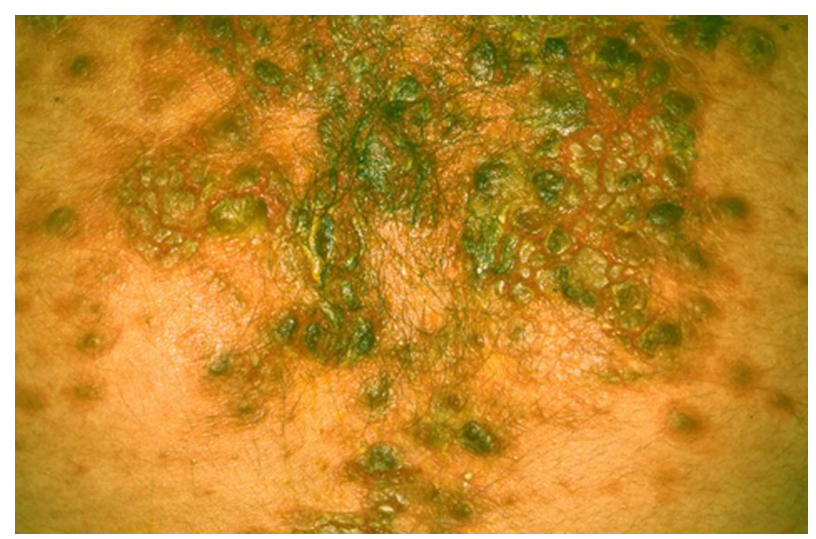

Figure I Crusted lesions involving seborrheic areas of the chest. medications apart from atorvastatin, so a diagnosis of $\mathrm{PE}$ relapse possibly induced by atorvastatin intake was made. Atorvastatin was immediately suspended. Systemic treatment with deflazacort (90 mg daily) and hydroxychloroquine (400 mg daily) and topical treatment with clobetasol propionate (twice a day) resulted in a regression of the disease within 12 weeks. The systemic corticosteroid therapy was gradually reduced while hydroxychloroquine was still in use. ${ }^{5}$ The patient is currently controlling cholesterol levels only with diet and sport.

\section{Discussion}

Statins, also known as 3-hydroxy-3-methylglutaril-CoA reductase inhibitors, are a class of hypolipidemic drugs that are very effective at lowering low-density lipoprotein (LDL) cholesterol with modest effects on lowering triglycerides and raising high-density lipoprotein (HDL) cholesterol. They are among the most prescribed drugs in the world for the important role in both the primary and secondary prevention of coronary heart disease, myocardial infarction, stroke, and peripheral artery disease. Statins are widely considered as safe but some serious adverse effects, such as myositis, myopathy, and rhabdomyolysis can rarely occur. In the last years, statins have often been associated with drug-induced reactions, including systemic lupus erythematosus, subacute cutaneous lupus erythematosus, dermatomyositis, polymyositis, ${ }^{6}$ and generalized eczematous skin rash. ${ }^{7} \mathrm{Six}$ cases of spiny keratoderma in patients treated with statins have been described. ${ }^{8}$ There are some reports about induction of alopecia by statins. ${ }^{8}$

Pravastatin can induce porphyria cutanea tarda, phototoxicity in the UVB range, and lichenoid drug eruptions. Simvastatin is reported to induce cheilitis, generalized exanthematous pustulosis, chronic actinic dermatitis, radiation reaction recall, and occupational contact dermatitis. Atorvastatin is reported to induce linear immunoglobulin A (IgA) bullous dermatosis, and toxic epidermal necrolysis. ${ }^{8}$

To the best of our knowledge, no case of pemphigus induced by statins has been reported in literature until now, but an interesting case of statin-induced lichen planus pemphigoides $^{9}$ and a case of pemphigus associated with an over-the-counter drug containing polyphenols have been described. ${ }^{10}$

The exact mechanism of statin-induced autoimmune reactions is unclear. Cellular apoptosis could be involved, in fact, statins are potent pro-apoptotic agents. ${ }^{1-13}$ The release of nuclear antigens from apoptotic cells into the circulation may evoke the production of pathogenic autoantibodies. 
The same mechanism involving the release of nuclear antigens might be implicated with other environmental factors such as UV radiation, which is a well-known triggering factor in systemic lupus erythematosus, subacute cutaneous lupus erythematosus, and dermatomyositis. Apoptosis could have a similar mechanism in the induction of pemphigus. In fact, a dysregulation of the normal programmed cell death of epidermal keratinocytes and/or deficient and inadequate clearance of apoptotic material (specifically desmoglein) may render it antigenic with the consequent production of autoantibodies. In the last years, numerous significant reports have shown that Fas-mediated apoptosis may play a central role in the acantholytic phenomenon. ${ }^{14-16}$

By lowering cholesterol levels, statins can influence the response of T-lymphocytes to exogenous signals. In fact, cholesterol is present in high concentrations in some areas of cell membranes implicated in the activation of immune response, defined as lipid rafts. Therefore lipid rafts act as a platforms for the activation of immune cells. ${ }^{6}$ In light of this, statins could alter T-lymphocyte behavior resulting in the release of forbidden clones.

Other evidence suggests that statins may directly modulate T-lymphocyte activation by promoting Th2 bias with consequent activation of B-lymphocytes and production of pathogenic autoantibodies, ${ }^{6}$ possibly including those responsible for pemphigus.

\section{Conclusion}

We found the case of our patient interesting because the disease re-appeared after many years, in a clinically more limited form but accompanied by an increase of the ANA titer, probably due to the concomitant use of atorvastatin.

Further studies are needed to evaluate the real role of statins in the induction of autoimmune diseases including pemphigus. Therefore, the increasing number of severe, sometimes fatal reactions to these drugs call for very careful pharmacovigilance, because of the well-known and less known side effects of statins, which are currently largely prescribed worldwide.

\section{Disclosure}

There are no funding sources. The authors have no conflict of interest or competing interests to disclose.

\section{References}

1. Senear FE, Usher B. An unusual type of pemphigus. Arch Dermatol. 1926;13:761-781.

2. Malik M, Ahmed AR. Concurrence of systemic lupus erythematosus and pemphigus: coincidence or correlation? Dermatology. 2007;214(3):231-239.

3. Baroni A, Puca RV, Aiello FS, et al. Cefuroxime-induced pemphigus erythematosus in a young boy. Clin Exp Dermatol. 2009;34(6): 708-710.

4. Ruocco V, Ruocco E, Lo Schiavo A, et al. Pemphigus: etiology, pathogenesis, and inducing or triggering factors: facts and controversies. Clin Dermatol. 2013;31(4):374-381.

5. Ruocco E, Wolf R, Ruocco V, et al. Pemphigus: associations and management guidelines: facts and controversies. Clin Dermatol. 2013;31(4):382-390.

6. Noël B. Lupus erythematosus and other autoimmune diseases related to statin therapy: a systematic review. J Eur Acad Dermatol Venereol. 2007;21(1):17-24.

7. Krasovec M, Elsner P, Burg G. Generalized eczematous skin rash possibly due to HMG-CoA reductase inhibitors. Dermatology. 1993;186(4):248-252.

8. Jowkar F, Namazi MR. Statins in dermatology. Int J Dermatol. 2010;49(11):1235-1243.

9. Stoebner PE, Michot C, Ligeron C, et al. Lichen plan pemphigoïde induit par la simvastatine. [Simvastatin-induced lichen planus pemphigoides]. Ann Dermatol Venereol. 2003;130(2 Pt 1): 187-190. French.

10. Lo Schiavo A, Brunetti G, Puca RV, Sangiuliano S, Ruocco E. Statinat-induced pemphigus: over-the-counter drugs too may be harmful! J Eur Acad Dermatol Venereol. 2009;23(1):70-71.

11. Baetta R, Donetti E, Comparato C, et al. Proapoptotic effect of atorvastatin on stimulated rabbit smooth muscle cells. Pharmacol Res. 1997;36(2):115-121.

12. Blanco-Colio LM, Villa A, Ortego M, et al. 3-Hydroxy-3-methylglutaryl coenzyme A reductase inhibitors, atorvastatin and simvastatin, induce apoptosis of vascular smooth muscle cells by downregulation of Bcl-2 expression and Rho A prenylation. Atherosclerosis. 2002;161(1):17-26.

13. Wibaut-Berlaimont V, Randi AM, Mandryko V, et al. Atorvastatin affects leukocyte gene expression in dyslipidemia patients: in vivo regulation of hemostasis, inflammation and apoptosis. J Thromb Haemost. 2005;3(4):677-685.

14. Wolf R, Matz H, Ruocco E, Ruocco V. The putative role of apoptosis in the induction of pemphigus. Med Hypotheses. 2005;64(1):44-45.

15. Puviani M, Marconi A, Cozzani E, Pincelli C. Fas ligand in pemphigus sera induces keratinocyte apoptosis through the activation of caspase-8. J Invest Dermatol. 2003;120(1):164-167.

16. Baroni A, Buommino E, Paoletti I, et al. Pemphigus serum and captopril induce heat shock protein 70 and inducible nitric oxide synthase overexpression, triggering apoptosis in human keratinocytes. $\mathrm{Br} \mathrm{J}$ Dermatol. 2004;150(6):1070-1080.

\section{Publish your work in this journal}

Drug Design, Development and Therapy is an international, peerreviewed open-access journal that spans the spectrum of drug design and development through to clinical applications. Clinical outcomes, patient safety, and programs for the development and effective, safe, and sustained use of medicines are a feature of the journal, which

\section{Dovepress}

has also been accepted for indexing on PubMed Central. The manuscript management system is completely online and includes a very quick and fair peer-review system, which is all easy to use. Visit http://www.dovepress.com/testimonials.php to read real quotes from published authors.

Submit your manuscript here: http://www.dovepress.com/drug-design-development-and-therapy-journal 